BEAUFILS, Anne-France; Louchet, Chantal - Écriture collaborative en cours de langue étrangère : ...

Para lá da tarefa: implicar os estudantes na aprendizagem de línguas estrangeiras no ensino superior. Porto: FLUP, 2019, pp. 68-94 DOI: https://doi.org/10.21747/9789898969217/paraa4

\title{
Écriture collaborative en cours de langue étrangère : une expérience entre des étudiants universitaires en France et au Portugal
}

\author{
Anne-France Beaufils \\ Universidade Católica Portuguesa, Centro de Estudos Comunicação e Cultura \\ (CECC), Lisbonne, Portugal \\ abeaufils@fch.lisboa.ucp.pt \\ Chantal Louchet \\ Universidade Católica Portuguesa, Centro de Estudos Comunicação e Cultura \\ (CECC), Lisbonne, Portugal \\ chantallouchet@fch.ucp.lisboa.pt
}

\begin{abstract}
Résumé
Cet article présente une expérience réalisée avec deux classes d'étudiants de l'Université Catholique Portugaise (niveaux A2 et B1), Lisbonne, Portugal et une classe d'étudiants de l'Université Paul Valéry (niveau A2), Montpellier, France. L'étude a pour but d'observer dans quelle mesure l'écriture collaborative peut avoir un impact sur l'apprentissage et sur la motivation des étudiants. Le logiciel FramaPad a été utilisé avec des étudiants des deux pays, les uns apprenant la langue des autres.

L'approche qualitative a été choisie et les données ont été recueillies par un questionnaire que les participants ont rempli. Les données ont ensuite été analysées selon les techniques d'analyse de contenu.

L'écriture collaborative, au même titre que d'autres outils relevant des TIC, apparaît comme un moyen approprié pour renforcer l'implication et la motivation des étudiants, mais également pour promouvoir l'apprentissage, étant donné que les étudiants ont la possibilité d'être créatifs et interagir avec leurs pairs dans une approche d'enseignement/apprentissage centrée sur l'apprenant.
\end{abstract}

Mots-clés : Éducation, TICE, écriture collaborative, motivation, apprentissage des langues 
BEAUFILS, Anne-France; Louchet, Chantal - Écriture collaborative en cours de langue étrangère : ... Para lá da tarefa: implicar os estudantes na aprendizagem de línguas estrangeiras no ensino superior. Porto: FLUP, 2019, pp. 68-94 DOI: https://doi.org/10.21747/9789898969217/paraa4

\begin{abstract}
This article presents an experiment conducted with two groups of students, from the Universidade Católica Portuguesa (A2 and B1 levels), Lisbon, Portugal and from the Paul Valéry University (A2 level), Montpellier, France. The study aims at observing to what extent collaborative writing can have some impact on students' learning and motivation. FramaPad was the software used with the students of both countries, each group learning the other group's language.

The qualitative approach was chosen and data were collected through a questionnaire participants filled in. Data were then analysed according to content analysis techniques. Collaborative writing, together with other ICT tools appears to be an appropriate way not only to reinforce students' involvment and motivation but also to promote learning, since students can be creative and interact with their peers in a learning/teaching student-based approach.
\end{abstract}

Keywords: Education, ICT, collaborative writing, motivation, language learning

\title{
1 - Introduction
}

A l'ère où les technologies occupent une place croissante dans la vie quotidienne des gens, y compris dans celle des étudiants, le défi que les enseignants doivent relever maintenir la motivation des étudiants, qui sont de plus en plus sollicités et prêts à se disperser - est plus que jamais d'actualité.

Notre étude s'efforce de mettre en avant l'impact que les techniques collaboratives en ligne, notamment la pratique d'écriture, dans une classe de langues en contexte universitaire, peuvent avoir sur l'apprentissage de la langue et sur la motivation des étudiants.

La question de recherche est donc formulée ainsi : l'utilisation, en cours de langue dans un contexte universitaire, d'un outil d'écriture collaborative en ligne, tel que FramaPad, permet-elle d'accroître la motivation des étudiants et consolider les apprentissages en langue étrangère ?

Une première partie sera consacrée à la question du choix et de la justification du projet : l'inclusion des TICE dans la classe de langues, les facteurs motivationnels et les caractéristiques de l'apprentissage en groupe. Dans un deuxième temps, le contexte et la méthodologie adoptée seront présentés. Ensuite, l'expérience sera décrite, en commençant par une présentation de l'outil FramaPad, suivie du déploiement et 
BEAUFILS, Anne-France; Louchet, Chantal - Écriture collaborative en cours de langue étrangère : ...

Para lá da tarefa: implicar os estudantes na aprendizagem de línguas estrangeiras no ensino superior.

Porto: FLUP, 2019, pp. 68-94

DOI: https://doi.org/10.21747/9789898969217/paraa4

déroulement du projet, ainsi que les difficultés rencontrées. Les résultats obtenus seront ensuite exposés et nous terminerons par la discussion de ces résultats.

\section{2 - Choix et justification du projet}

\section{1 - Inclusion des TICE dans la classe de langues}

Les recherches des deux dernières décennies concernant l'impact des TICE sur l'apprentissage des langues font apparaître la technologie comme un facteur motivationnel. De plus, par ce biais, les processus de mémorisation seraient facilités et l'accès au matériel et à la communication authentiques rendu possible (Zhao, 2003). Si les technologies en général ont, de façon consensuelle, un effet sur les réactions affectives des apprenants, augmentant leur motivation, certains chercheurs soulignent que les effets sur l'apprentissage doivent encore être démontrés (Golonka, Bowles, Frank, Richardson, \& Freynik, 2014).

Les recherches de ces auteurs, en reprenant les travaux de Sykes (2005), tendent à démontrer que, parmi les technologies utilisées dans l'apprentissage des langues, le chat écrit est celle qui permet d'augmenter de façon la plus significative la quantité mais aussi la complexité de la production langagière de l'apprenant. Cet élément intéresse notre étude dans la mesure où l'écriture collaborative peut être rapprochée du chat de par certains aspects comme la synchronicité (même si, dans notre cas, les classes accédaient et modifiaient le document les unes après les autres), les participants multiples et le support. Ce résultat pourrait être dû, selon Sykes (2005), au délai qui existe dans les interactions écrites de type chat. En effet, les participants disposent de plus de temps pour élaborer leurs phrases, construire et donc pratiquer des structures complexes, que dans le chat oral et la discussion orale. Le chat écrit pourrait aussi amener les étudiants à se centrer davantage sur la forme.

Dans ce contexte, l'enseignant doit adapter son enseignement de manière à utiliser les TICE de la façon la plus efficace possible. Il devient médiateur. D'après Michel (2001), il se transforme en chef d'orchestre.

\section{2 - Facteurs motivationnels}

Notre étude est ancrée sur les travaux de Dortier (2017), qui identifie trois éléments de réponse à la question "Peut-on motiver autrui? ». Le premier, l'environnement, considéré un puissant moteur de motivation, est, dans notre cas, la classe d'étudiants, le groupe. Le second, l'attrait de la nouveauté, réside dans l'expérience elle-même, nouvelle pour tous les étudiants. Enfin, le troisième, la réussite, était, dans une certaine 
BEAUFILS, Anne-France; Louchet, Chantal - Écriture collaborative en cours de langue étrangère : ...

Para lá da tarefa: implicar os estudantes na aprendizagem de línguas estrangeiras no ensino superior.

Porto: FLUP, 2019, pp. 68-94

DOI: https://doi.org/10.21747/9789898969217/paraa4

mesure, garantie, puisqu'il n'y avait pas d'évaluation ni de possibilité de « rater ».

Un autre élément mérite aussi d'être pris en compte : la confiance en soi. Du fait que les étudiants étaient amenés à travailler ensemble, au sein de leur classe et avec deux autres classes, on peut, à la suite de Galand (2016), penser que la comparaison entre leurs propres performances et celles des autres étudiants, a eu un impact sur la confiance en eux, un des piliers de l'apprentissage (Grangeat \& Vygotski, 2016).

\section{3 - L'apprentissage en groupe}

Dans son article, Grangeat (2016) reprend la primauté que Vygotski a donné à l'apprentissage social en distinguant une Zone Proximale de Développement (ZPD) : écart entre ce que l'apprenant peut faire seul (développement actuel) et ce qu'il peut faire avec l'aide d'autrui (capacité potentielle de développement). L'apprentissage est rendu possible si la médiation sociale intervient dans cette ZPD. C'est pourquoi les interactions sociales sont importantes dans la construction des processus cognitifs et l'acquisition des connaissances.

Bandura (2007) a également souligné l'influence du contexte social sur les apprentissages. L'individu est capable d'accroître ses compétences en observant et imitant le comportement d'autres personnes du groupe, qui sont valorisées et servent de modèle et d'inspiration. C'est la théorie du modelage social. Le groupe apparaît donc comme lieu privilégié d'apprentissage, sa cohésion étant un facteur d'efficacité.

La psychologie sociale met aussi en avant la notion d'apprentissage coopérant (capacités d'apprentissage résultant des interactions au sein du groupe), liée à la pédagogie de groupe. Les interactions - échanges de points de vue, discussions, propositions, argumentation, mises en cause - entraînent à elles seules un progrès collectif, que ce soit dans la compréhension d'un problème, la recherche de solutions, ou même l'acquisition d'un savoir. C'est dans ce cadre que s'inscrit l'écriture collaborative.

\section{3 - Méthodologie}

Trois groupes d'apprenants, chacun ayant son enseignant, ont été mis en contact : deux classes d'étudiants de l'Université Catholique Portugaise, Lisbonne, Portugal, et une classe d'étudiants de l'Université Paul Valéry, Montpellier, France.

Les étudiants du Portugal (neuf étudiants de deuxième année et huit étudiants de troisième année de licence de LEA - Langues Etrangères Appliquées) apprenaient le français tandis que les étudiants de France (quinze étudiants de première année de 
BEAUFILS, Anne-France; Louchet, Chantal - Écriture collaborative en cours de langue étrangère : ...

Para lá da tarefa: implicar os estudantes na aprendizagem de línguas estrangeiras no ensino superior.

Porto: FLUP, 2019, pp. 68-94

DOI: https://doi.org/10.21747/9789898969217/paraa4

licence LLCER - Langue, Littérature, Civilisation Etrangère et Régionale - Portugais et LEA Portugais/Anglais) apprenaient le portugais. Chacun(e) devant s'exprimer dans la langue qu'il/elle étudie.

Le scénario pédagogique a été défini par les trois enseignants, qui ont choisi un thème d'actualité, le développement durable, auquel sera subordonnée la production écrite, dans le but de comparer la réalité de chaque pays et de chaque université.

La comparaison et le partage des connaissances sur le thème du développement durable avaient aussi pour but de permettre de croiser les regards sur un même thème, d'apprécier, de commenter, de critiquer, d'ouvrir les horizons et favoriser en même temps un échange linguistique entre les deux pays.

Les données ont été recueillies par le biais d'un questionnaire anonyme que les étudiants ont rempli à la fin du semestre : quatorze questions pour lesquelles ils devaient cocher une réponse sur une échelle de 1 à 5, trois questions ouvertes et une question ayant pour réponse oui / non.

L'analyse des données a été faite autour de deux grands axes: l'impact de l'expérience sur la motivation et son impact sur l'apprentissage, ou plus précisément sur le ressenti des étudiants quant à d'éventuels progrès accomplis grâce à l'expérience.

L'expérience n'avait jamais été mise en place auparavant. Aussi, les enseignants ontils fait le choix d'une durée très courte : chaque classe devait y consacrer deux cours de $90 \mathrm{~min}$, de façon à ce que, en cas d'échec, l'impact sur le déroulement des cours au long du semestre soit limité.

\section{4 - Expérience}

\section{1 - Présentation de l'outil FramaPad}

II s'agit d'un service en ligne de traitement de texte simplifié, qui permet la production à plusieurs. C'est un outil simple, facilement accessible, librement utilisable, souvent utilisé dans l'éducation. II suffit de créer un Pad (un éditeur de texte collaboratif en ligne), ce qu'a fait notre collègue de l'université de Montpellier, à la suite de quoi les utilisateurs peuvent commencer à rédiger leur texte. Chaque étudiant-participant est identifié par une couleur, ce qui permet de savoir qui est en train d'écrire et quels sont les étudiants qui participent. Chaque contribution apparaît à l'écran en temps réel et est enregistrée au fur et à mesure de l'élaboration du texte. Pour cela, le document collaboratif qui est en cours est visualisé d'une part de forme collective sur l'écran de la salle de classe, projeté par le vidéoprojecteur (poste de l'enseignant) et, d'autre part, de forme individuelle par chaque étudiant, sur son propre ordinateur ou smartphone, permettant 
BEAUFILS, Anne-France; Louchet, Chantal - Écriture collaborative en cours de langue étrangère : ... Para lá da tarefa: implicar os estudantes na aprendizagem de línguas estrangeiras no ensino superior. Porto: FLUP, 2019, pp. 68-94 DOI: https://doi.org/10.21747/9789898969217/paraa4

la saisie individualisée des données. Les étudiants sont tous connectés sur le document en même temps. Pour garder sa couleur, l'étudiant doit utiliser le même ordinateur (le sien) à chaque séance. Le texte en cours est en permanence accessible à tout à chacun, les étudiants connectés peuvent donc à tout moment effectuer des changements tels que corriger l'orthographe d'un mot, finir une phrase ou une idée, rajouter un connecteur.

\section{2 - Déploiement et déroulement du projet}

Étant donné le peu de séances imparties (deux séances de 90 minutes pour chaque classe), il a été demandé aux étudiants de faire une recherche au préalable sur le thème afin d'accélérer le processus de production écrite ; le but n'étant pas d'être à la recherche d'idées mais de les structurer assez rapidement et de façon cohérente en un texte.

Rappelons qu'une production de texte suppose la maîtrise d'un certain nombre de points sur le plan du lexique, des conjugaisons, de la syntaxe et des aspects grammaticaux notamment.

Résumons notre démarche pédagogique en deux phases.

Première phase :

recherches au préalable sur le thème évoqué (individuellement et hors contexte de cours) ;

mise en commun des différentes idées ;

écriture ;

correction collective (orthographe / structure des phrases / conjugaison / vocabulaire etc.) par les étudiants eux-mêmes, l'enseignant n'intervenant qu'à leur demande.

Deuxième phase :

lecture et compréhension des textes réponses ;

puis, à nouveau, écriture.

On retrouve dans notre démarche les « cinq grandes catégories du processus interactif de l'enseignement et de l'apprentissage : (se) motiver, (s') informer, (s') activer, interagir, produire » (Lebrun, 2007, p. 39).

\section{3 - Difficultés rencontrées}

Certaines des difficultés rencontrées ont été d'ordre technique. Le nombre excessif d'accès par rapport à la capacité de la bande passante a engendré des blocages, 
BEAUFILS, Anne-France; Louchet, Chantal - Écriture collaborative en cours de langue étrangère : ...

Para lá da tarefa: implicar os estudantes na aprendizagem de línguas estrangeiras no ensino superior.

Porto: FLUP, 2019, pp. 68-94

DOI: https://doi.org/10.21747/9789898969217/paraa4

notamment sur le campus de Lisbonne, que nous avons dépassés en créant un nombre très restreint de groupes de travail ( 3 ou 4 ), chacun ayant un seul accès.

À Montpellier, il n'y a pas ou peu eu de difficultés de connexion, ce qui a permis des interactions nombreuses et souples, plusieurs d'entre eux travaillant pour cette occasion à partir de leur téléphone portable - interdit habituellement en cours. Ainsi, le fait que durant ces séances ils ont été incités à les utiliser a contribué à développer leur intérêt pour l'exercice et les a poussés à interagir par écrit davantage qu'ils ne le font habituellement à l'oral. Certains se sont même attribué naturellement des tâches spécifiques durant les séances, comme par exemple celle de relire et de corriger les fautes d'orthographe de leurs collègues, autant de prises d'initiative qui ont été valorisées par l'enseignant.

Un étudiant de l'université française a pourtant noté « les bugs informatiques et le temps de réaction du logiciel qui met longtemps à réagir " mais ils sont neuf de l'université portugaise à avoir mentionné les problèmes techniques, notamment la mauvaise connexion wifi ou l'instabilité du système. Un étudiant suggère d'essayer avec une autre plate-forme et un autre encore estime que pouvoir interagir en temps réel serait intéressant. Néanmoins, un étudiant a apprécié la structure de FramaPad, un autre a apprécié le logiciel « parce que [...] c'est une très belle manière de se confronter avec des classes de pays différents » et un troisième a aimé les couleurs et les dialogues simultanés.

Le choix du thème n'a pas recueilli l'unanimité des étudiants. Ils sont deux de l'université portugaise à exprimer leur désir de choisir eux-mêmes le thème, et un qui aimerait essayer avec différents sujets la prochaine fois. Toutefois, un étudiant a « aimé pouvoir échanger sur des sujets qui sont importants à notre époque », un autre estime que «c'est un sujet important pour notre vie » et un troisième a trouvé que « les sujets choisis étaient intéressants et d'actualité ».

La gestion des tours de parole au sein du groupe, aussi bien lors de la phase de préparation, phase essentielle pour la réussite de l'expérience, que lors du déroulement, a engendré certaines difficultés. Cette pratique d'écriture, la co-écriture, demande une planification commune de la part des groupes et également au sein de chaque groupe, dans le but de produire un texte avec des idées cohérentes. Or les étudiants voulaient souvent participer en même temps. Chacun a dû trouver sa place et sentir à quel moment il devait participer, quelles idées il devait développer, s'il pouvait avoir des idées de nature divergente et de quelle manière il pouvait les introduire sans générer de conflits. Accepter les idées des autres est absolument nécessaire à la création du texte 
BEAUFILS, Anne-France; Louchet, Chantal - Écriture collaborative en cours de langue étrangère : ...

Para lá da tarefa: implicar os estudantes na aprendizagem de línguas estrangeiras no ensino superior.

Porto: FLUP, 2019, pp. 68-94

DOI: https://doi.org/10.21747/9789898969217/paraa4

mais aussi au maintien des bonnes relations entre les étudiants. Comme le dit Paul Ricœur, " la tolérance n'est pas une concession que je fais à l'autre, elle est la reconnaissance du principe qu'une partie de la vérité m'échappe » (Ricœur, 1988, p. 134). Un étudiant a précisément mentionné « [...] parfois, d’autres étudiants ont des opinions étranges, c'est difficile de collaborer totalement les uns avec les autres ».

Certaines difficultés provenant du travail de groupe ont aussi été mises en avant, notamment l'impatience de quelques étudiants : « Je n'aime pas quelque ma collègue se sent impatiente quand une ou un collègue ne parle pas bien, exprime pas bien en français », "Le problème dans ce type de travail c'est qu'il y a généralement toujours des étudiants qui prennent sans cesse la parole, ne laissant pas forcément le temps aux autres personnes de réfléchir et de réellement participer». De plus, il y a parfois "beaucoup d'idées désorganisées et de répétitions ».

La gestion du temps, quant à elle, a été délicate, en conséquence du choix qui avait été fait de ne consacrer que deux cours d'une heure et demie au projet, ce qui a créé une certaine pression. Trois étudiants l'ont regretté : «Peut-être le peu de temps que nous avions pour pouvoir faire telle activité. Je pense que ce serait bien d'avoir un peu plus de temps pour des activités novatrices et très intéressantes comme celle-ci ». "Ce serait bien que l'exercice dure plus longtemps, sur plusieurs semaines, par exemple. », «Ma suggestion c'est de le faire durer plus longtemps».

Le blocus de l'université de Montpellier au second semestre 2018 n'a pas été oublié. Un étudiant regrette « de ne pas avoir été plus loin à cause du blocus »1.

\section{5 - Résultats}

Notre méthode se veut, dans un premier temps, descriptive et interprétative (5.1), au travers de l'observation des étudiants pendant les deux cours qui ont eu lieu, puis évaluative (5.2), au travers du questionnaire final dont les réponses aux questions ouvertes ont été analysées suivant les techniques d'analyse de contenu (Bardin, 2007).

\section{1 - Observation de l'attitude des étudiants en cours}

L'expérience a démontré combien la collaboration était porteuse d'interaction entre les étudiants : les échanges oraux sont fréquents entre eux, l'enseignant n'étant qu'un médiateur. Le rôle de celui-ci est avant tout de promouvoir la diffusion des idées entre les étudiants et de les mettre par écrit. La maîtrise de l'écrit passe ici par un véritable

\footnotetext{
1 Manifestation des étudiants et enseignants contre le projet de réforme de l'accès à l'enseignement supérieur en France (Parcours Sup), particulièrement violente à Montpellier.
} 
BEAUFILS, Anne-France; Louchet, Chantal - Écriture collaborative en cours de langue étrangère : ...

Para lá da tarefa: implicar os estudantes na aprendizagem de línguas estrangeiras no ensino superior.

Porto: FLUP, 2019, pp. 68-94

DOI: https://doi.org/10.21747/9789898969217/paraa4

dialogue de réflexion entre les étudiants, créant une dynamique de classe différente. Les termes relatifs au travail d'équipe au sein de la classe et avec les autres classes ont été mentionnés par plusieurs étudiants dans les réponses à la question ouverte «Qu'est-ce qui vous a le plus plu? »:

« collaboration/contribuer [...] pour un résultat commun/travail en groupe/avec la classe/en équipes 》 (11 fois) ;

« découvrir/connaître/entendre/lire/partager des idées/opinions » (11 fois) ;

" échanger/converser/communiquer/parler/interagir » (11 fois).

L'expérience a également démontré combien la collaboration a été porteuse de qualité. Une volonté de «bien faire », de «faire bonne impression », de montrer son savoir à l'autre a eu des répercussions très positives sur l'ambiance de la classe et sur les étudiants eux-mêmes, engendrant un véritable travail d'équipe, où la prise en compte des différents points de vue a été facilement acceptée. II n'y a pas eu de conflits générés par des idées de nature divergente, ce qui a facilité la tâche des enseignants. Les apports de chaque étudiant ont été valorisés ; puis une aide mutuelle s'est naturellement établie pour retranscrire dans la langue d'apprentissage l'idée proposée par l'un d'entre eux.

Les enseignants ont pu observer l'implication des étudiants dans la thématique choisie, leur engagement et leur responsabilité. Les étudiants se concentrent et sont motivés pour «ne pas faire d'erreurs ». Quatre étudiants ont précisément évoqué les erreurs: "C'était plus intéressant pour corriger notre "erros"», «[...] et la correction m'ont le plus plu», «[...] voir les possibles erreurs», «Essayer de rédiger des phrases parfaites $»^{2}$. Le dialogue étant ouvert entre les étudiants, un partage de commentaires s'est naturellement instauré visant à la «perfection» du texte. Par exemple, comment dit-on "ambiente" en français? Le mot «environnement» s'écrit avec un «n» ou deux «n»? Attention! Au passé composé avec l'auxiliaire être, il y a accord, il faut que tu ajoutes un e au participe passé, etc. Les enseignants ont été témoins d'une interaction orale accrue entre les élèves lors de ces sessions. Comme l'indique Lebrun (2007, p. 14), « au niveau de la pédagogie active [...], c'est l'activité mentale de l'apprenant qui est mise en évidence, qui est mise à l'épreuve ».

Cette expérience a permis de mettre l'accent sur l'autonomie des apprenants, leur conférant un espace d'interaction beaucoup plus ample qu'à l'habituel, résultant d'échanges constants avec leur entourage, ce qui favorise l'apprentissage, comme nous l'avons vu plus haut, si cet échange se situe dans la Zone Proximale de Développement

\footnotetext{
2 Les réponses des étudiants ont été transcrites telles quelles.
} 
BEAUFILS, Anne-France; Louchet, Chantal - Écriture collaborative en cours de langue étrangère : ...

Para lá da tarefa: implicar os estudantes na aprendizagem de línguas estrangeiras no ensino superior. Porto: FLUP, 2019, pp. 68-94 DOI: https://doi.org/10.21747/9789898969217/paraa4

(Vygotski, 2003). Dans ce contexte précis, les étudiants sont devenus « des sujets actifs au lieu de récepteurs d'un savoir transmis » (Michel, 2001, p. 385).

Nous avons constaté, par le biais de cette expérience, que l'écriture collaborative, dans un but précis d'élaborer un texte en commun, mobilise des compétences de plusieurs ordres, notamment, la pratique d'écriture, les échanges oraux, la lecture et la compréhension des écrits, mais aussi informationnelles, numériques et promeut également la collaboration, la tolérance, l'écoute, la discipline et le travail en équipe.

De plus, elle offre la possibilité de mettre en place des projets pédagogiques centrés sur l'étudiant comme acteur de son savoir, de sa connaissance et de ses apprentissages en langue. «L'apprenant est sur la scène » (Michel, 2001, p. 390).

L'authenticité situationnelle - il s'agit d'une tâche ancrée dans la vie réelle et non pas d'une situation de simulation en classe «fictionnelle »-, ce contexte bidirectionnel (Portugal / France), a intensifié les relations entre les divers participants. Les destinataires de l'acte communicatif (le texte produit) sont les étudiants étrangers, ceux qui apprennent leur langue maternelle. Parmi les aspects qui leur a le plus plu, deux étudiants de l'université française ont fait référence à cette authenticité : « Le fait de converser avec de vrais élèves. Cela a apporté un but concret à notre travail. », « [...] d'échanger avec de réels étudiants portugais ».

\section{2 - Résultats obtenus}

Les données ont été recueillies à travers le questionnaire rempli par les étudiants à l'issue de l'expérience. Les résultats et analyses présentés ci-dessous se fondent sur l'analyse quantitative et qualitative des réponses des étudiants, recueillies par questionnaire par rapport à trois grands axes: l'impact de l'expérience sur l'apprentissage, l'impact de l'expérience sur la motivation et les découvertes et échanges interculturels. 
BEAUFILS, Anne-France; Louchet, Chantal - Écriture collaborative en cours de langue étrangère : ... Para lá da tarefa: implicar os estudantes na aprendizagem de línguas estrangeiras no ensino superior.

\subsection{1 - Impact de l'expérience sur la motivation}

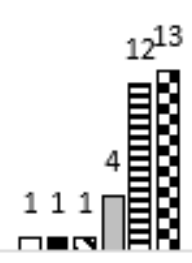

a. Collaborer avec vos collègues de cours

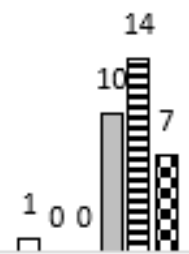

b. Produire votre propre texte

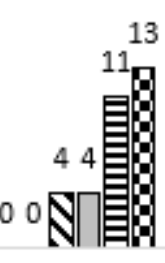

c. Lire les

réponses des étudiants des autres classes

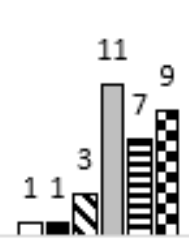

d. Corriger les autres étudiants

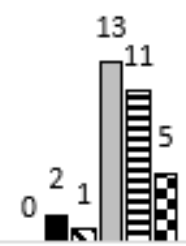

e. Faire une recherche sur le thème

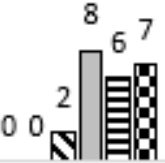

f. Découvrir / g. Autre(s) utiliser le Iogiciel

FramaPad

Sachant que $1=$ pas du tout et $5=$ beaucoup

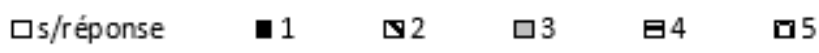

Figure 1. Nombre de réponses des étudiants pour l'ensemble des trois classes 1. Vous avez aimé a., b., c., d., e., f., g.

Les 3 points qui ont recueilli le plus grand nombre de réponses cochées «4» ou «5», correspondant aux aspects de l'expérience que les étudiants ont le plus aimé sont :

- «Collaborer avec vos collègues de cours» (25 étudiants sur un total de 32 ont coché le 4 ou le 5);

- «lire les réponses des étudiants des autres classes» (24 étudiants sur 32 ont coché le 4 ou le 5);

- «produire votre propre texte» (21 étudiants sur 32 ont coché le 4 ou le 5). 
BEAUFILS, Anne-France; Louchet, Chantal - Écriture collaborative en cours de langue étrangère : ... Para lá da tarefa: implicar os estudantes na aprendizagem de línguas estrangeiras no ensino superior.

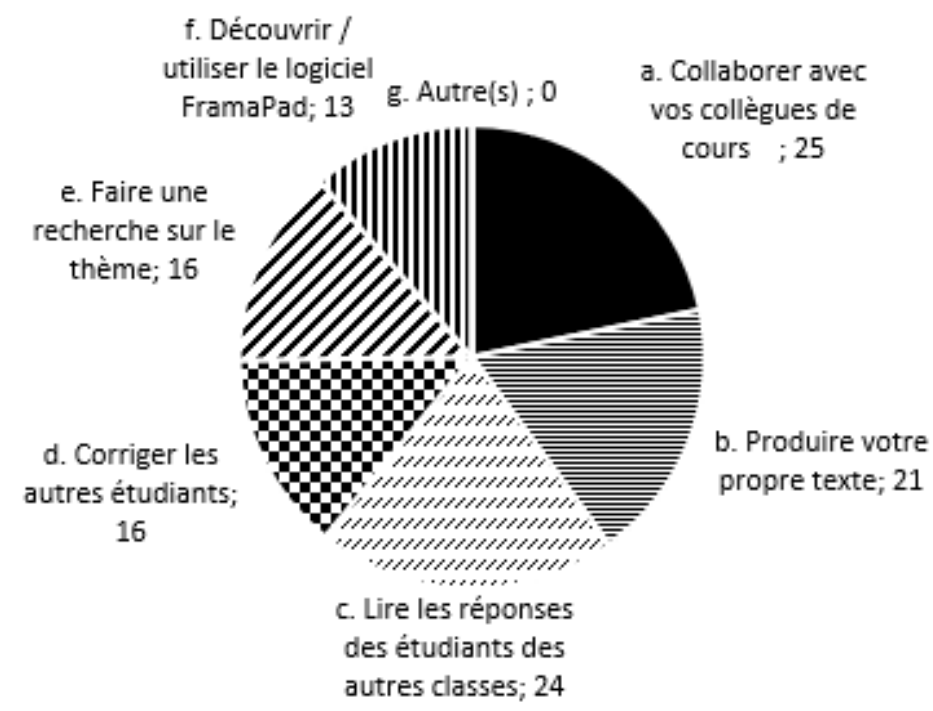

Figure 2. Nombre de réponses des étudiants pour l'ensemble des trois classes (réponses 4 et 5) 1. Vous avez aimé a., b., c., d., e., f.

Les 3 points qui ont recueilli le plus grand nombre de réponses cochées «1» ou «2», correspondant aux aspects de l'expérience que les étudiants ont le moins aimé sont :

- «lire les réponses des étudiants des autres classes» (4 étudiants sur 32 ont coché le 1 ou le 2);

- «corriger les autres étudiants» (4 étudiants sur 32 ont coché le 1 ou le 2);

- «faire une recherche sur le thème » (3 étudiants sur 32 ont coché le 1 ou le 2). 
BEAUFILS, Anne-France; Louchet, Chantal - Écriture collaborative en cours de langue étrangère : ... Para lá da tarefa: implicar os estudantes na aprendizagem de línguas estrangeiras no ensino superior.

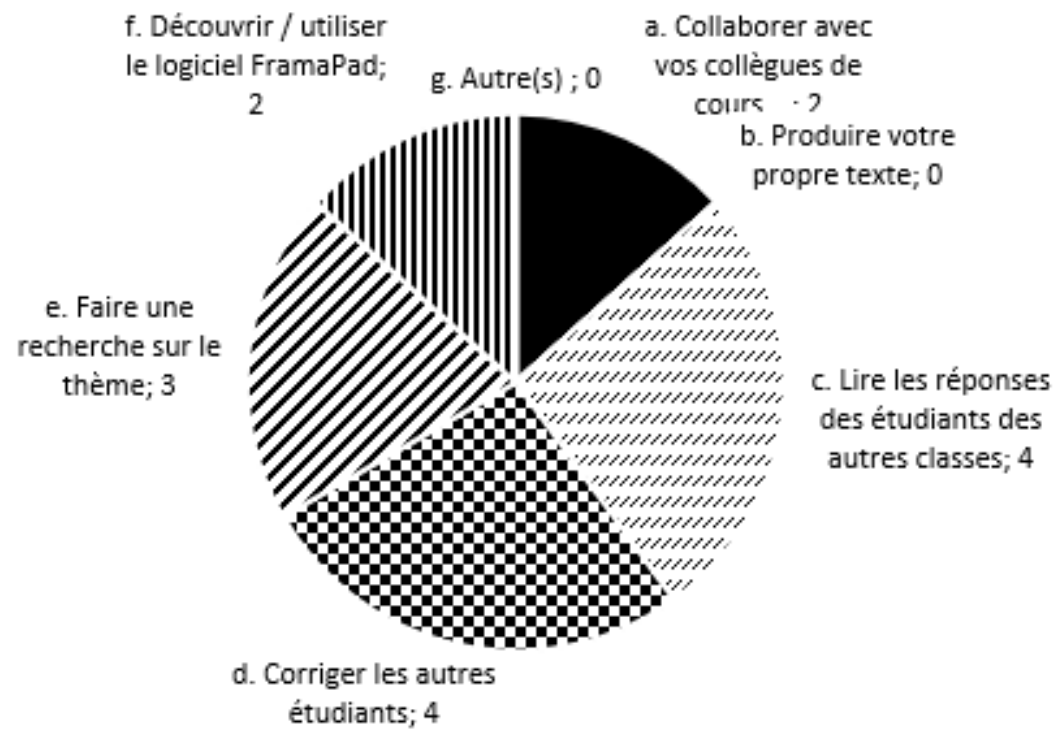

Figure 3. Nombre de réponses des étudiants pour l'ensemble des trois classes (réponses 1 et 2) 1. Vous avez aimé a., b., c., d., e., f, g.

Les 3 points qui ont recueilli le plus grand nombre de réponses cochées « 3 », correspondant aux aspects de l'expérience que les étudiants ont moyennement aimé sont:

«faire une recherche sur le thème » (13 étudiants sur 32 ont coché le 3);

- «corriger les autres étudiants» (11 étudiants sur 32 ont coché le 3);

- «produire votre propre texte» (10 étudiants sur 32 ont coché le 3). 
BEAUFILS, Anne-France; Louchet, Chantal - Écriture collaborative en cours de langue étrangère : ... Para lá da tarefa: implicar os estudantes na aprendizagem de línguas estrangeiras no ensino superior.

Porto: FLUP, 2019, pp. 68-94 DOI: https://doi.org/10.21747/9789898969217/paraa4

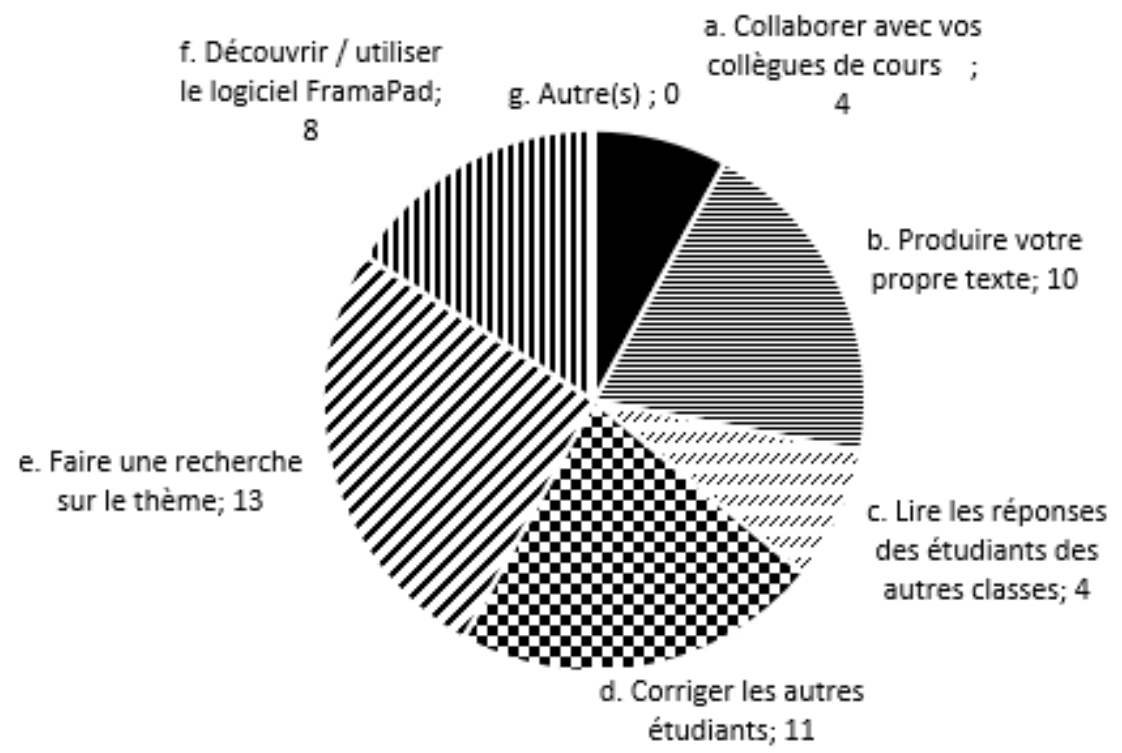

Figure 4. Nombre de réponses des étudiants pour l'ensemble des trois classes (réponse 3) 1. Vous avez aimé a., b., c., d., e., f, g.

Pour ce qui est de l'aspect «lire les réponses des étudiants des autres classes», il apparaît que plus de la moitié des étudiants de l'université française ont coché le 5 (beaucoup aimé), par rapport à un peu moins d'un tiers chez étudiants de l'université portugaise. Ces derniers ont davantage aimé «collaborer avec vos collègues de cours» (7 étudiants).

Les réponses à la question «Voudriez-vous renouveler l'expérience?» sont aussi un indicateur de la satisfaction globale des étudiants. Les étudiants de l'université de Montpellier ont tous répondu «oui». Les 5 réponses négatives proviennent d'une étudiante portugaise et de 4 étudiants macanais, de l'Université Catholique de Lisbonne.

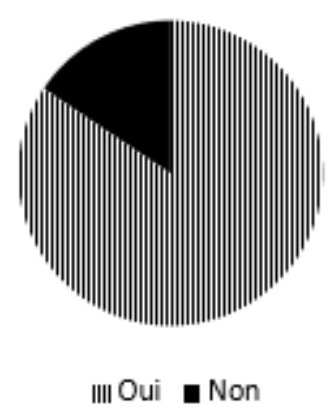

Figure 5. Nombre de réponses des étudiants pour l'ensemble des trois classes 6 . Voudriezvous renouveler l'expérience? 
BEAUFILS, Anne-France; Louchet, Chantal - Écriture collaborative en cours de langue étrangère : ... Para lá da tarefa: implicar os estudantes na aprendizagem de línguas estrangeiras no ensino superior.

Un étudiant de Montpellier suggère « que cette activité se répète plus souvent dans un avenir ».

\subsection{2 - Impact de l'expérience sur l'apprentissage}

II s'agit de l'analyse du ressenti des étudiants quant à la consolidation des apprentissages et éventuels progrès accomplis grâce à l'expérience.

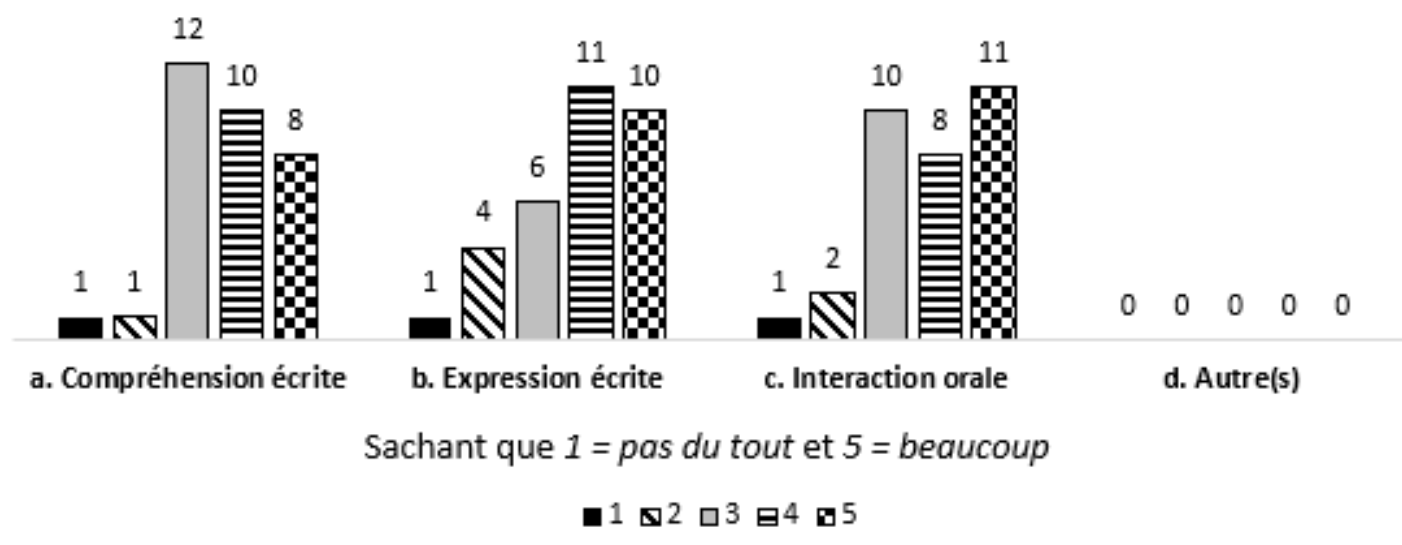

Figure 6. Nombre de réponses des étudiants pour l'ensemble des trois classes 2. Cette expérience vous a-t-elle aidé à développer vos compétences en termes de a., b.,c., d.

II n'y a pas de grande variation dans la perception que les étudiants ont des compétences que l'expérience a le plus aidé à développer. Presque deux tiers des étudiants ( 18 à 21 sur 32 ) ont coché « 4 » ou « 5 » pour les 3 aspects proposés. Selon eux, l'expérience a beaucoup contribué à développer la compréhension et l'expression écrites ainsi que l'interaction orale. 
BEAUFILS, Anne-France; Louchet, Chantal - Écriture collaborative en cours de langue étrangère : ... Para lá da tarefa: implicar os estudantes na aprendizagem de línguas estrangeiras no ensino superior.

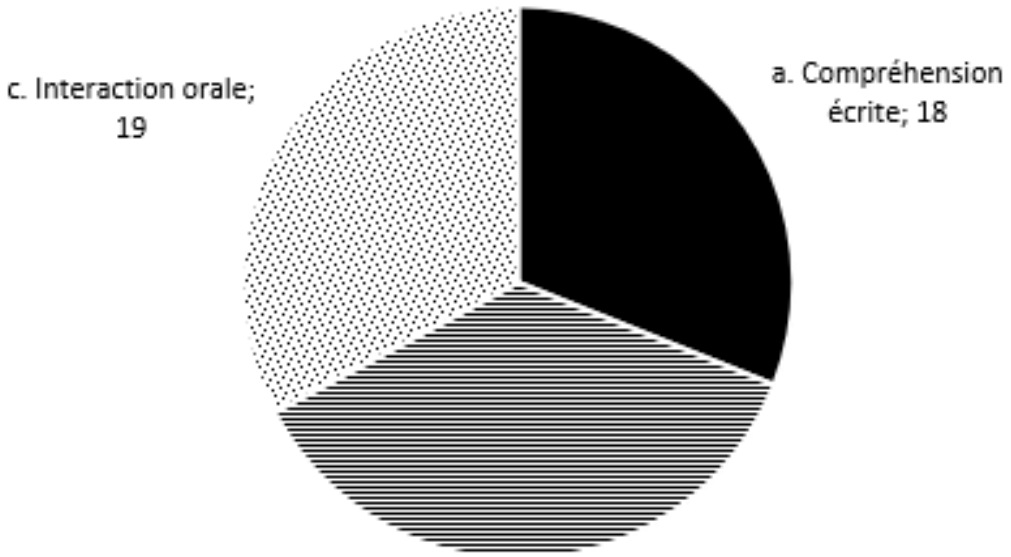

b. Expression écrite;

21

Figure 7. Nombre de réponses des étudiants pour l'ensemble des trois classes 2. Cette expérience vous a-t-elle aidé à développer vos compétences en termes de (réponses 4 ou 5) : a., b., c.

Ils ne sont qu'entre 6 et 10, selon les critères, à estimer que l'expérience a moyennement contribué (réponses «3») au développement de ces compétences.

Seulement quatre étudiants de l'université de Lisbonne et trois de Montpellier pensent qu'elle n'a pas du tout ou peu contribué (réponses «1» ou «2») à leur développement.

Pour être plus précis, en ce qui concerne le développement de l'expression écrite, deux étudiants ont coché «2» (Montpellier et Lisbonne).

Pour ce qui est du développement de l'interaction orale, un étudiant de Montpellier a coché «1» et trois de Lisbonne ont coché «2», dont une qui s'exprime très volontiers malgré ses difficultés, et deux macanaises, timides, qui n'ont pas réussi à s'imposer.

Quant à la compréhension écrite, 1 étudiant de Montpellier a coché «1». 
BEAUFILS, Anne-France; Louchet, Chantal - Écriture collaborative en cours de langue étrangère : ... Para lá da tarefa: implicar os estudantes na aprendizagem de línguas estrangeiras no ensino superior.

Porto: FLUP, 2019, pp. 68-94

DOI: https://doi.org/10.21747/9789898969217/paraa4

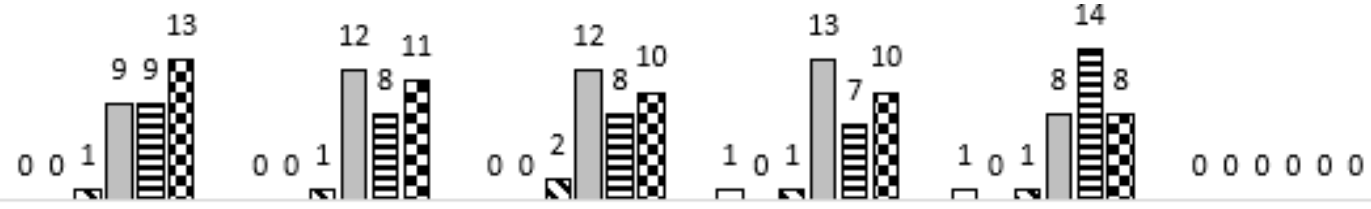

$$
\begin{aligned}
& \text { a. Etendre votre b. Consolider vos c. Améliorer la d. Exposer vos e. Revoir certains f. Autre(s) } \\
& \text { vocabulaire conjugaisons syntaxe idées } \begin{array}{c}
\text { aspects } \\
\text { grammaticaux }
\end{array} \\
& \text { Sachant que } 1=\text { pas } d u \text { tout et } 5=\text { beaucoup }
\end{aligned}
$$

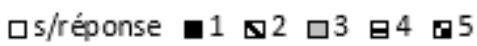

Figure 8. Nombre de réponses des étudiants pour l'ensemble des trois classes 3. Cette expérience a-t-elle été utile pour a., b., c., d., e., f.

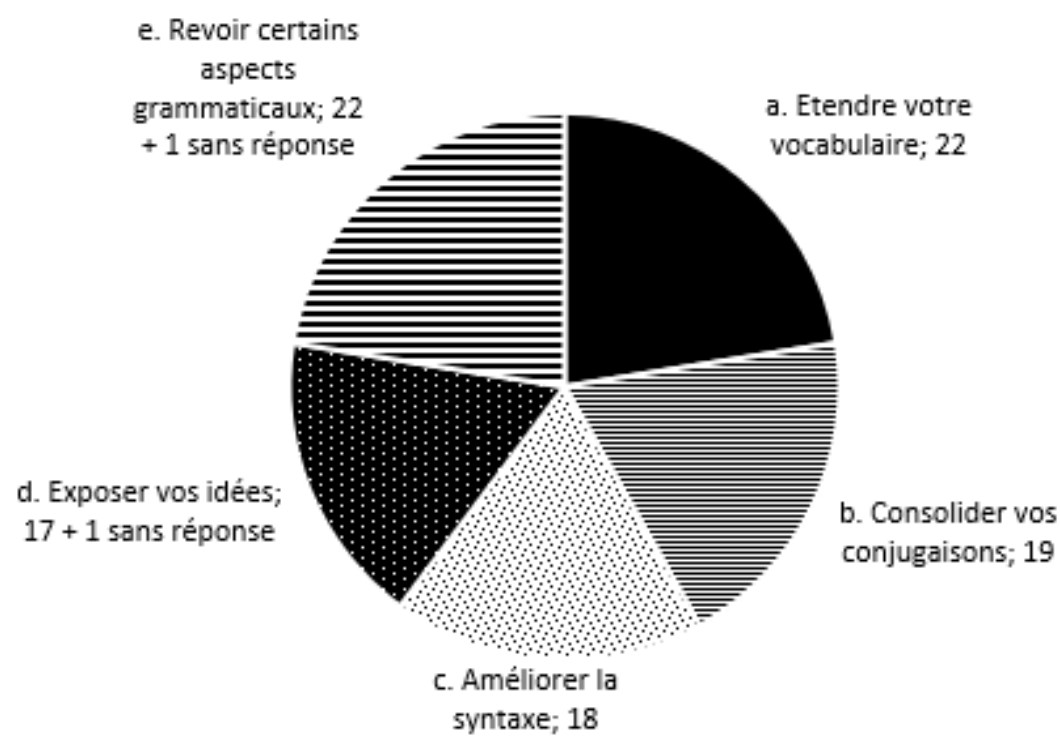

Figure 9. Nombre de réponses des étudiants pour l'ensemble des trois classes 3. Cette expérience a-t-elle été utile pour a., b., c., d., e. (réponses 4 ou 5) 
BEAUFILS, Anne-France; Louchet, Chantal - Écriture collaborative en cours de langue étrangère : ... Para lá da tarefa: implicar os estudantes na aprendizagem de línguas estrangeiras no ensino superior.

Porto: FLUP, 2019, pp. 68-94 DOI: https://doi.org/10.21747/9789898969217/paraa4

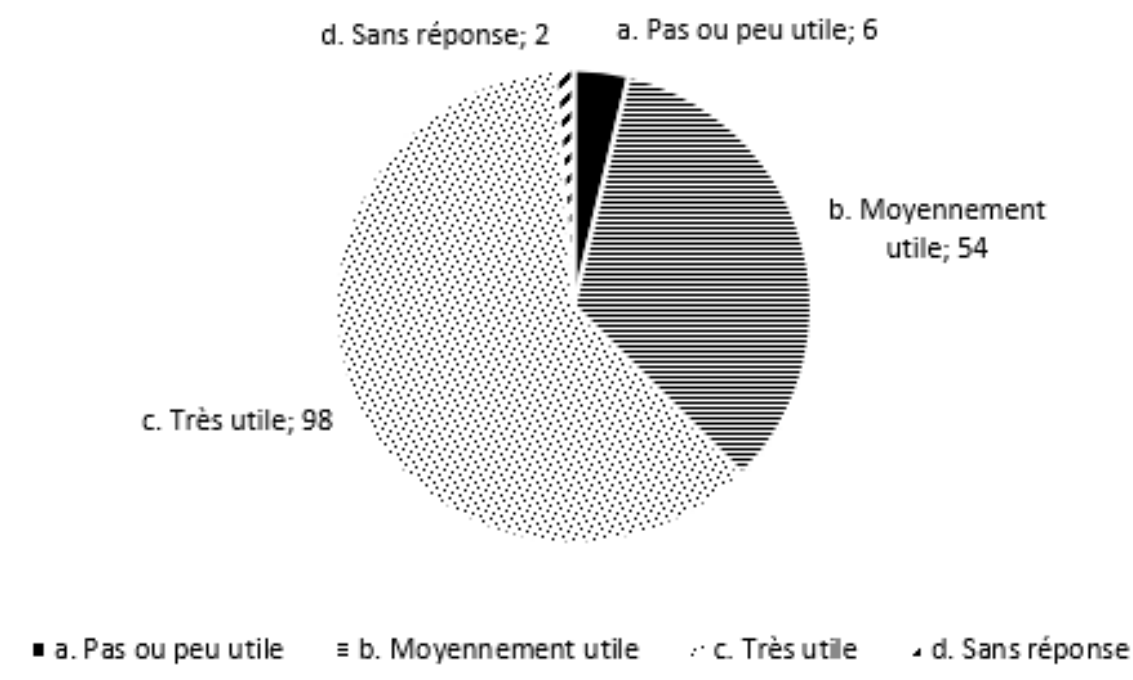

Figure 10. Nombre de réponses des étudiants pour l'ensemble des trois classes Utilité pour la consolidation des apprentissages langagiers.

II n'y a que trois étudiants, de l'université française, qui ont considéré que l'expérience n’a pas ou peu été utile, en ce qui concerne trois points:

pour exposer ses idées (1 étudiant a coché «2»);

pour améliorer la syntaxe (deux autres ont coché «2»);

et pour revoir certains aspects grammaticaux (1 étudiant a coché «2»).

Certains commentaires des étudiants à la question ouverte «Qu'est-ce qui vous a le plus plu?» ont fait allusion aux aspects langagiers: «Pour nous améliorer notre technique d'écriture ", " me rendre compte de mes capacités de compréhension écrite. Et apprendre du vocabulaire pour structurer une idée ou une réponse », «Cela permet [...] de pouvoir s'améliorer sans forcément faire des exercices de conjugaisons 》.

La compréhension orale a même été citée par un étudiant: «Acho que foi um exercício prático que pode melhorar a capacidade de compreensão oral, compreensão escrita e gramática » (Je pense que c'était un exercice pratique qui peut améliorer la capacité de compréhension orale, compréhension écrite et grammaire).

\subsection{3 - Pré-requis}

II ressort de cette expérience que les compétences des apprenants sont à prendre en considération avant le début du projet. Ce genre d'exercice créatif exige un requis minimum de la langue d'apprentissage, A2, afin de réaliser les diverses tâches proposées. L'étudiant doit être « capable de produire de l'écrit dans une situation de 
BEAUFILS, Anne-France; Louchet, Chantal - Écriture collaborative en cours de langue étrangère : ...

Para lá da tarefa: implicar os estudantes na aprendizagem de línguas estrangeiras no ensino superior.

Porto: FLUP, 2019, pp. 68-94

DOI: https://doi.org/10.21747/9789898969217/paraa4

communication donnée et apporter une remédiation aux écrits qu'il produit. Les ressources lexicales et grammaticales font parties de ces outils » (Gerbault, 2010, p. 40).

L'expérience semble avoir plus profité aux meilleurs étudiants. La réponse d'un étudiant de France à la question «Qu'est-ce qui vous a déplu/dérangé»est la suivante : «La partie orale, seulement parce que j'ai encore du mal à comprendre et à m'exprimer à l'oral». II serait donc utile donc reformuler l'expérience pour l'adapter à ceux qui ont plus de difficultés, par exemple faire travailler les étudiants en binôme pour éviter que les étudiants les plus faibles ne soient seuls.

\subsection{4 - Découvertes et échanges interculturels}

Les réponses des étudiants à la question ouverte «Qu'est-ce qui vous a le plus plu ? " donnent une place prépondérante à l'aspect interculturel, mentionné par la moitié d'entre eux. L'un indique la richesse de l'échange au sein de sa classe «entre nous premièrement mais aussi entre les étudiants de l'Université de Lisbonne ».

Les échanges avec des étudiants de l'université de l'autre pays sont valorisées par plusieurs étudiants "communiquer avec d'autres étudiants en langue à l'étranger», «partager les idées et lire les réponses (surtout celles des étudiants de Montpellier)», «[...] pouvoir converser avec des étudiants tel que nous dans un autre pays et une autre langue», «avoir pu correspondre avec des étudiants en portugais en France», «échanger avec de réels étudiants portugais», "parler avec des étudiants français et connaître leurs idées», "c'est une très belle manière de se confronter avec des classes de pays différents».

Cette «confrontation» a permis à certains une prise de conscience en ce qui concerne l'apprentissage d'une langue étrangère dans un autre pays: «[...] Voir que les étudiants étrangers parlent rapidement notre langue et constater qu'ils apprennent plus rapidement que nous à maîtriser les langues étrangères».

L'ouverture vers un autre monde est aussi un aspect que certains étudiants ont relevé : «[...] parler et [...] échanger des idées avec les étudiants de autres universités. C'était très intéressant de percevoir la vie quotidienne des étudiants dans d'autres universités et leurs systèmes et la gestion de leurs ressources», "elle t'aide à connaître plus la situation actuelle d'autres pays et d'enrichir tes connaissances grâce à ceux d'autres étudiants», "avoir des avis externes d'autres personnes et leurs ressentis sur ce qu'ils voient et ce qui se passe en France. Voir d'autres types d'universités et d'autres paysages», «[...] la possibilité de prendre contact facilement avec les étudiants d'une 
BEAUFILS, Anne-France; Louchet, Chantal - Écriture collaborative en cours de langue étrangère : ...

Para lá da tarefa: implicar os estudantes na aprendizagem de línguas estrangeiras no ensino superior.

Porto: FLUP, 2019, pp. 68-94

DOI: https://doi.org/10.21747/9789898969217/paraa4

université de l'autre pays et de pouvoir connaître différentes opinions sur le sujet, ou de son université».

Certains y ont vu davantage de portes s'ouvrant pour l'avenir: «[...] le contact avec d'autres élèves d'universités pour que nous puissions créer de plus en plus de ponts qui augmentent nos connaissances académiques, culturelles et visions de mondes différents, avec des personnes qui sont dans la même situation que la nôtre [...] parce que notre avenir dépend de ponts et bonne volonté mutuelles», «[...] cela donne aussi un aperçu de ce qu'est leur université si l'un d'entre nous serait tenté d'y faire 1 ou 2 semestres en Erasmus».

\section{6 - Discussion}

Les réponses des étudiants seront analysées à la lumière des travaux de Vygotski, qui a mis en évidence l'importance des interactions sociales dans l'acquisition des connaissances (Grangeat \& Vygotski, 2016).

II ressort que l'échange est l'aspect le plus fréquemment mentionné comme le plus positif de l'expérience dans la mesure où il s'est produit avec d'autres étudiants, comme eux, de «vrais» étudiants, dans un cadre authentique. Ils ont communiqué sur un sujet commun, ce qui leur a permis de connaître la réalité d'un autre pays et voir quelles pratiques existent ailleurs; deux tiers d'entre eux ont en effet aimé, voire beaucoup aimé, collaborer avec leurs propres collègues de cours et lire les réponses des étudiants des autres classes. Pour communiquer, ils ont utilisé la langue d'apprentissage qui se trouvait être la langue maternelle des étudiants de l'autre pays. Ce croisement a permis un enrichissement mutuel. Ils ont pu appréhender leurs difficultés langagières et celles de leurs pairs. La prise de conscience des étudiants s'est donc produite à plusieurs niveaux, sur le fond et sur la forme: la moitié des étudiants ont aimé ou beaucoup aimé «corriger les autres étudiants». Rappelons, cf. 2.2, que l'observation par les étudiants de la réussite ou de l'échec de leurs pairs peut jouer un rôle sur la confiance en eux, influencée par la comparaison de leurs performances avec celles des autres (Galand, 2016). C'est peut-être une des raisons pour lesquelles la plupart des étudiants ont aimé ou beaucoup aimé ces deux points: ils ont acquis davantage de confiance en euxmêmes. Cette corrélation mériterait d'être confirmée par une étude plus spécifique.

La comparaison des résultats entre les deux pays révèle une plus grande curiosité de la part des étudiants de l'université française. Plus de la moitié ont beaucoup aimé lire les réponses des étudiants des autres classes, contre un peu moins d'un tiers chez les étudiants de l'université portugaise. L'extrait d'un courriel du professeur le renforce: 
BEAUFILS, Anne-France; Louchet, Chantal - Écriture collaborative en cours de langue étrangère : ...

Para lá da tarefa: implicar os estudantes na aprendizagem de línguas estrangeiras no ensino superior. Porto: FLUP, 2019, pp. 68-94 DOI: https://doi.org/10.21747/9789898969217/paraa4

«Mes élèves, appréhensifs au départ, ont finalement bien aimé cette production textuelle. Ils attendent impatiemment la suite... J'ai pu faire travailler mes étudiants normalement aujourd'hui, ils ont été très intéressés et motivés par nos échanges».

Les 5 réponses négatives à la question «Voudriez-vous renouveler l'expérience?» proviennent d'une étudiante portugaise et de 4 étudiants macanais, de l'Université Catholique de Lisbonne. Tous les étudiants de l'université de Montpellier ont répondu «oui». Les enseignantes ont pu observer que les étudiants de Macao réagissent moins bien à la spontanéité. Ils ont besoin de plus de temps que les autres pour se préparer pour une tâche, poussés par leur fort désir de perfection, qui bloque parfois la fluidité des échanges. C'est peut-être une clé de lecture de ces réponses.

Si l'on observe les réponses des étudiants quant au développement de l'interaction orale, de la compréhension et expression écrites, par le biais de l'expérience, le bilan est donc clairement positif: entre la moitié et deux tiers des étudiants ont attribué «4» ou «5». En effet, lorsqu'ils découvrent les réponses des autres classes à leurs questions, les étudiants sont amenés à développer leurs compétences de lecture et de compréhension globales du message. "Aujourd'hui, avec la numérisation, le texte connaît une nouvelle époque: celle des supports dynamiques où la lecture rejoint "naturellement" l'écriture » (Stiegler, 1991, p. 122). Quant à l'interaction orale, il s'agit des discussions au sein de la classe. C'est donc l'ensemble des compétences qui est développé. La notion d'apprentissage coopérant, abordée au point 2.2., prend tout son sens ici, les interactions entraînant le progrès collectif.

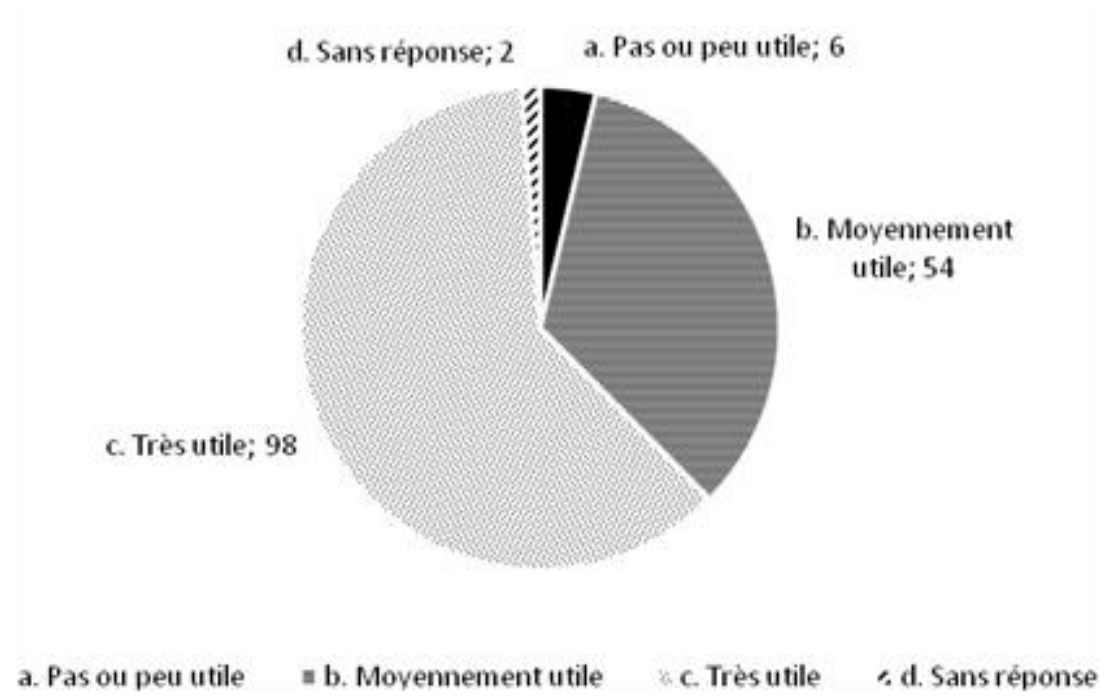

Figure 11. Nombre de réponses des étudiants pour l'ensemble des trois classes Utilité pour la consolidation des apprentissages langagiers 
BEAUFILS, Anne-France; Louchet, Chantal - Écriture collaborative en cours de langue étrangère : ... Para lá da tarefa: implicar os estudantes na aprendizagem de línguas estrangeiras no ensino superior.

En ce qui concerne l'utilité pour la consolidation des aspects langagiers, nous pourrions nous attendre à ce que les réponses «1» (pas ou peu utile) ou «2» (moyennement utile) proviennent des étudiants bilingues, pour qui il serait légitime que l'expérience soit inutile sur ces points-là, mais cela n'a pas été le cas. Les meilleurs étudiants ont précisément répondu «3» ou «4».

Parmi les facteurs qui pourraient expliquer ces résultats, nous pouvons évoquer la personnalité des étudiants, la culture(les étudiants macanais, comme mentionné plus haut, ont souvent des difficultés à travailler dans la spontanéité, il leur faut du temps de préparation), ou leur bagage langagier trop faible pour suivre la marche.

En France, plus qu'au Portugal, où le système de sélection à l'entrée à l'Université est différent, certains étudiants viennent en cours sans réelle motivation, surtout en première année de licence. Ceci expliquerait donc le faible intérêt manifesté par un ou deux étudiants.

Une autre difficulté qui apparaît dans les questionnaires est celle d'exposer ses idées. On constate ainsi que le travail proposé peut revêtir une difficulté à laquelle on ne pense pas immédiatement, qui est celle d'avoir des idées arrêtées sur un sujet. Par ailleurs, certains étudiants, de par leur personnalité et leur manque de confiance en eux, peinent à considérer leurs idées comme recevables et intéressantes, et préfèrent s'abstenir de les exprimer.

Enfin, on constate à Montpellier une différence culturelle majeure entre les étudiants français du groupe et les trois étudiantes Erasmus espagnoles, qui ont toutes un excellent niveau de langue et ont considéré, malgré cela, que l'activité collaborative avait été très intéressante pour elles du point de vue de l'acquisition de compétences, tout comme du point de vue de la thématique et de l'intérêt de «produire collectivement» un texte. Ceci dit, les 15 étudiants souhaitent renouveler l'expérience.

\section{7 - Conclusion}

L'expérience a eu, pour les étudiants des deux pays, et selon eux, un impact positif sur l'apprentissage ainsi que sur les échanges interculturels. De ce fait, la motivation s'en est trouvée renforcée, ce qui confirme notre hypothèse.

En effet, cinq étudiants seulement sur 32 ne souhaitent pas renouveler l'expérience, dont, parmi eux, quatre macanais, pour qui, comme nous l'avons vu, il est souvent plus difficile d'agir dans la spontanéité et de s'exposer. 
BEAUFILS, Anne-France; Louchet, Chantal - Écriture collaborative en cours de langue étrangère : ...

Para lá da tarefa: implicar os estudantes na aprendizagem de línguas estrangeiras no ensino superior.

Porto: FLUP, 2019, pp. 68-94

DOI: https://doi.org/10.21747/9789898969217/paraa4

II semblerait aussi, en reprenant la métaphore de l'orchestre (Michel, 2001), que les musiciens (étudiants) doivent avoir un bagage langagier minimal (pré requis A2) pour que l'orchestre puisse jouer sans fausse note. En revanche, contrairement aux attentes des enseignants, les meilleurs étudiants ont estimé que l'expérience leur a été très profitable pour développer leurs compétences d'interaction orale, compréhension et expression écrites. Les étudiants dans leur ensemble ont souligné comme positifs le fait d'avoir pu appréhender leurs difficultés et celles de leurs pairs, et ont aimé corriger les autres étudiants.

L'impact positif de l'expérience sur la motivation apparaît dans les réponses des étudiants qui mentionnent les échanges interculturels avec de « vrais étudiants» (aspect authentique), la confrontation avec des classes de pays différents ayant permis une prise de conscience quant à l'apprentissage d'une langue étrangère dans un autre pays, l'ouverture vers un autre monde, des portes s'ouvrant pour l'avenir et la création de ponts.

L'enthousiasme de certains étudiants est notable dans leurs suggestions, qu'ils soient de l'université portugaise ou française. Les étudiants de l'université portugaise ont notamment mentionné: «Travailler avec plus d'étudiants et plus d'universités», «Faire un projet Ecriture Collaborative pendant tout le semestre avec différents sujets», "Ce serait amusant si, au-delà de l'écriture collaborative en cours et correspondance en commun, il y avait la possibilité de communiquer « un à un» avec les collègues de France », «[...] visiter chaque université avec la classe et les professeurs [...] avec la préparation d'un plan d'activités culturelles ». Ceux de l'université française ont indiqué : «Faire un débat simultané de deux pays différents, par exemple eux, du Portugal, et nous, de France», «Si le nombre d'étudiants le permet, attribuer un groupe de 3 étudiants français à 3 étudiants portugais», «Peut-être il serait bien d'organiser une activité qui consistait en que chaque élève français parle avec un élève portugais via Skype ou par e-mail», "Si c'est possible, pourquoi ne pas essayer de contacter les correspondants en vidéo pour approfondir davantage l'expression et la compréhension orale».

Néanmoins, il peut être utile de rappeler la mise en garde de Gray (2008): lorsque la technologie prend place en classe de langue, il faut veiller à ce que les objectifs pédagogiques et les moyens non technologiques gardent la première place. La technologie ne peut, en aucun cas, remplacer/compenser un enseignement. C'est un outil, au service d'une pédagogie. 
BEAUFILS, Anne-France; Louchet, Chantal - Écriture collaborative en cours de langue étrangère : ...

Para lá da tarefa: implicar os estudantes na aprendizagem de línguas estrangeiras no ensino superior.

Porto: FLUP, 2019, pp. 68-94

DOI: https://doi.org/10.21747/9789898969217/paraa4

\section{Références bibliographiques}

Bandura, A. (2007). L'autoefficacité (2nd ed.). Louvain-la-Neuve: De BoeckSupérieur.

Bardin, L. (2007). L'analyse de contenu. Paris: PUF.

Dortier, J. (2017). Motivation, la liste de nos envies. In La motivation (pp. 9-20). Editions Sciences Humaines.

Galand, B. (2016). Réussite scolaire et estime de soi. In Eduquer et former (pp. 159164). Auxerre: Editions Sciences Humaines.

Gerbault, J. (2010). TIC : panorama des espaces d'interaction et de rétroaction pour l'apprentissage de l'écriture en langue étrangère. Revue Française de Linguistique Appliquée, XV, 37-52. Retrieved from https://www.cairn.info/revue-francaise-delinguistique-appliquee-2010-2-page-37.htm.

Golonka, E. , Bowles, A. , Frank, V. , Richardson, D. , \& Freynik, S. (2014). Technologies for foreign language learning: a review of technology types and their effectiveness. Computer Assisted Language Learning, 70-105. https://doi.org/10.1080/09588221.2012.700315

Grangeat, M., \& Vygotski, L. (2016). L'apprentissage par le groupe. In Eduquer et former (pp. 134-141). Auxerre: Editions Sciences Humaines.

Gray, L. (2008). Effective practice with e-Portfolios. Bristol: UK: JISC Innovation Group. Retrieved from http://www.ssphplus.info/files/effective_practice_e-portfolios.pdf

Lebrun, M. (2007). Théorie et méthodes pédagogiques pour enseigner et apprendre. Quelle place pour les TIC dans l'éducation ? Louvain-la-Neuve: De Boeck.

Michel, G. (2001). Repenser la pédagogie grâce aux TIC dans l'enseignement initial à l'université. In E. dir. Delozanne \& P. Jacobini (Eds.), Sciences et techniques éducatives (pp. 379-410.). Retrieved from https://www.persee.fr/issue/stice_12651338_2001_num_8_3

Ricoeur, P. (1988). Tolérance, intolérance, intolérable. Bulletin de La Société de I'histoire Du Protestantisme Français, 134, 435-452. Retrieved from https://www.jstor.org/stable/24296238

Stiegler, B. (1991). Annotation, navigation, édition électroniques: vers une géographie de la connaissance. Linx, Hors-Série $N^{\circ} 4$, Texte et o, 121-131. Retrieved from https://www.persee.fr/doc/linx_0246-8743_1991_hos_4_1_1191

Sykes, J. (2005). Synchronous chat and pragmatic development: Effects of oral and written chat. Calico Journal, 22, 399-431. Retrieved from https://pdfs.semanticscholar.org/0520/b0a4be35901b24a0da2d0e43c53e0a47384c.pdf

Vygotski, L. (2003). Pensamento e linguagem. São Paulo: Martins Fontes. Retrieved 
BEAUFILS, Anne-France; Louchet, Chantal - Écriture collaborative en cours de langue étrangère : ... Para lá da tarefa: implicar os estudantes na aprendizagem de línguas estrangeiras no ensino superior. Porto: FLUP, 2019, pp. 68-94 DOI: https://doi.org/10.21747/9789898969217/paraa4

from http://www.ebooksbrasil.org/adobeebook/vigo.pdf

Zhao, Y. (2003). Recent developments in technology and language learning: A literature review and meta-analysis. Calico Journal, 21, 7-27. Retrieved from https://www.jstor.org/stable/24149478 
BEAUFILS, Anne-France; Louchet, Chantal - Écriture collaborative en cours de langue étrangère : ... Para lá da tarefa: implicar os estudantes na aprendizagem de línguas estrangeiras no ensino superior.

\section{Annexe 1}

Questionnaire réalisé auprès des élèves de l'Université Catholique Portugaise (2 classes de niveaux A2 et B1), Lisbonne, Portugal et de l'Université Paul Valéry (1 classe de niveau A2), Montpellier, France.

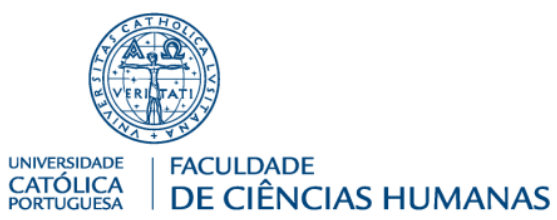

\section{QUESTIONNAIRE “Projet ECRITURE COLLABORATIVE”}

Nom du participant :

Vous répondrez aux questions 1 à 3 selon l'échelle suivante :

\begin{tabular}{|l|l|l|l|l|}
\hline 1 & 2 & 3 & 4 & 5 \\
\hline
\end{tabular}

Sachant que 1 = pas du tout et $5=$ beaucoup

1. Vous avez aimé :

a. Collaborer avec vos collègues de cours

b. Produire votre propre texte

c. Lire les réponses des étudiants des autres classes

d. Corriger les autres étudiants

e. Faire une recherche sur le thème

f. Découvrir / utiliser le logiciel FramaPad

\begin{tabular}{|r|r|r|r|r|}
\hline 1 & 2 & 3 & 4 & 5 \\
\hline 1 & 2 & 3 & 4 & 5 \\
\hline 1 & 2 & 3 & 4 & 5 \\
\hline
\end{tabular}

g. Autre(s) :

\begin{tabular}{|r|r|r|r|r|}
\hline 1 & 2 & 3 & 4 & 5 \\
\hline 1 & 2 & 3 & 4 & 5 \\
\hline 1 & 2 & 3 & 4 & 5 \\
\hline
\end{tabular}

2. Cette expérience vous a-t-elle aidé à développer vos compétences en termes de :

a. Compréhension écrite

b. Expression écrite

c. Interaction orale

d. Autre(s) :

\begin{tabular}{|r|r|r|r|r|}
\hline 1 & 2 & 3 & 4 & 5 \\
\hline 1 & 2 & 3 & 4 & 5 \\
\hline 1 & 2 & 3 & 4 & 5 \\
\hline
\end{tabular}


BEAUFILS, Anne-France; Louchet, Chantal - Écriture collaborative en cours de langue étrangère : ... Para lá da tarefa: implicar os estudantes na aprendizagem de línguas estrangeiras no ensino superior.

3. Cette expérience a-t-elle été utile pour :

a. Etendre votre vocabulaire

b. Consolider vos conjugaisons

c. Améliorer la syntaxe

d. Développer vos idées

e. Revoir certains aspects grammaticaux

f. Autre(s):

\begin{tabular}{|r|r|r|r|r|}
\hline 1 & 2 & 3 & 4 & 5 \\
\hline 1 & 2 & 3 & 4 & 5 \\
\hline 1 & 2 & 3 & 4 & 5 \\
\hline 1 & 2 & 3 & 4 & 5 \\
\hline 1 & 2 & 3 & 4 & 5 \\
\hline
\end{tabular}

4. Qu'est-ce qui vous a le plus plu ?

5. Qu'est-ce qui vous a dérangé / déplu ?

6. Voudriez-vous renouveler l'expérience ?

○ Oui

- Non

7. Vos suggestions :

Merci de votre collaboration. 